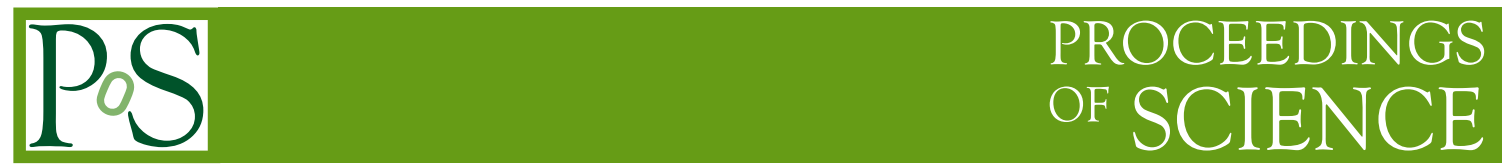

\title{
Top quark mass measurements at hadron colliders
}

\section{Gordon Watts*}

University of Washington

E-mail: gwatts@uw.edu

The top quark mass is the best known quark mass. This talk will be a quick review of the measurements of the top mass at the four main collider experiments, CDF and DZERO from the Tevatron accelerator located at Fermilab, and ATLAS and CMS from the Large Hadron Collider located at CERN. Besides results at brief survey of techniques and future prospects will be given.

Frontiers of Fundamental Physics 14 - FFP14,

15-18 July 2014

Aix Marseille University (AMU) Saint-Charles Campus, Marseille

* Speaker. 